Article

\title{
Information and Physics
}

\section{Vlatko Vedral $^{1,2}$}

1 Clarendon Laboratory, University of Oxford, Parks Road, Oxford OX1 3PU, UK;

E-Mail: v.vedral1@physics.ox.ac.uk; Tel.: +44-1865-272-389; Fax: +44-1865-272-375

2 Center for Quantum Technology, National University of Singapore, Singapore 117543, Singapore

Received: 6 March 2012; in revised form: 9 May 2012 / Accepted: 10 May 2012 /

Published: 11 May 2012

\begin{abstract}
In this paper I discuss the question: what comes first, physics or information? The two have had a long-standing, symbiotic relationship for almost a hundred years out of which we have learnt a great deal. Information theory has enriched our interpretations of quantum physics, and, at the same time, offered us deep insights into general relativity through the study of black hole thermodynamics. Whatever the outcome of this debate, I argue that physicists will be able to benefit from continuing to explore connections between the two.
\end{abstract}

Keywords: information; quantum; relativity

\section{Introduction}

For the Ancient Greeks, the route to the ultimate understanding of the universe was through geometry. "Let no one ignorant of geometry enter through my doors" stood written at the entrance to Plato's academy. Two millennia later, Kepler still believed that God was a geometer and even Newton, who originally undoubtedly developed his calculus in an algebraic way, still chose to write "Principia" in terms of geometry. This made the exposition more cumbersome, but he believed that more people would accept it that way as geometry was held in such high regard.

Newton, however, already put physics before geometry [1]. He famously said that straight lines are only meaningful because they are actually implemented in practice by freely moving bodies. In other words, physics defines geometry. This philosophy culminated with Einstein, whose general theory of relativity [2] is all about gravity actually being a particular kind of geometry of space and time. Massive bodies curve space-time, which in turn acts on them and influences their motion. Geometry and gravity are, in fact, synonymous in this grand vision of Einstein's. 
However, the whole philosophy changed dramatically shortly after the discovery of general relativity, when it became apparent that this is only half of the story as far as our understanding of the laws of physics. The other half is, of course, quantum physics, whose interpretation does not lend itself naturally to geometry. There is now a strong belief, instead, that the branch of mathematics most useful for understanding quantum physics is actually information theory (see, for example [3]).

\section{Quantum Physics and Information}

Quantum physics does, of course, have features that have a more geometric flavor (e.g., angles between physical states being related to their fidelity). And there have indeed been numerous attempts to fully geometrize quantum physics (see, e.g., [4]) in order to bring it closer to unification with gravity. None of them, however, achieved either the unification with gravity or a deeper understanding of the quantum.

Two features of quantum physics have a strong information theoretic flavor. One is the fact that states of physical systems are described by what Schrödinger called "catalogues of information". Namely, when we are talking about different states of a physical system, such as an electron, we have to give amplitudes for the electron to be located at each point in space and at any given time. These amplitudes, when mod squared, tell us about the probabilities for the electron to occupy these positions at given times (in other words, these probabilities will give us relative frequencies for detecting the electron in given positions, if we choose to do so).

The other feature is what Einstein called "a spooky action at a distance". In quantum physics, objects can be correlated to a much higher degree than anything in classical physics (e.g., in general relativity). Entanglement, as Schrödinger famously said, was (not just one of many, but) the characteristic trait of quantum mechanics [5]. One of its key experimental manifestations is in correlations that two entangled particles display when measured and these correlations exceed anything that classical physics allows [6]. Bell's inequalities were designed to quantify this excess of correlations [7]. Bell made two assumptions when deriving his inequalities more than forty years ago, that of "locality" and that of "reality". The first is meant to comply with the principles of special relativity, namely that no signal can travel faster than light. The second says that measurement outcomes should be determined independently of the measurement settings (i.e., there is a reality out there independent of us choosing how and what to measure). The fact that quantum mechanics has been experimentally verified to violate Bell's inequalities, forces us to drop either the locality of reality assumptions (or both!).

Entanglement is at odds with (general) relativity on both accounts of quantum non-locality. If something really propagates faster than light, then relativity is invalidated. If, on the other hand, properties do not exist independently of measurements, should the same be true of space and time? Should space and time also be created through our interactions and measurements rather than be a background arena in which events take place? 


\section{General Relativity and Information}

This brings us to a natural question. If quantum physics is best understood as a theory of information, can general relativity also be brought closer to information theory? That might help us bridge the gap between the quantum and relativistic aspects of reality. The relationship between information and general relativity has also had a rich and exciting history. The first inkling came from the black hole entropy and Bekenstein bound [8]. If a bit of information is written into an area of the size of Planck length squared, then the Black hole entropy is equal to the number of bits that can be written into its area [3]. This led to the development of black hole thermodynamics. But, if a black hole has entropy and temperature, then it must radiate, a process that could only be explained quantum mechanically and this was done by Hawking [9].

A real breakthrough, however, came from Jacobson [10]. In 1995, he published a letter in the Physical Review, where he argued that one can derive Einstein's field equations for gravity from thermodynamics itself. The key to Jacobson's logic was the Bekenstein relationship between entropy and area, which, as we mentioned, has a strong information-theoretic foundation.

It is very simple to explain how gravity might arise from entropy. I will use Newtonian gravity since it makes the whole argument simpler. First we acknowledge the fundamental thermodynamical relationship that entropy times temperature equals heat. Heat itself is nothing but a form of energy, which according to Einstein equals mass times the speed of light squared. The entropy of a region we assume to be proportional to its area, in other words radius squared. The temperature is, according to the works of Davies and Unruh [11], proportional to acceleration, which in turn is force divided by mass (from Newton's second law). Putting all this together gives us the force equal to the product of masses divided by distance squared, namely Newton's gravity! And that is, more or less, what is behind Jacobson's claim.

Jacobson's idea was (and still probably is) considered very controversial, because it might imply that all those physicists trying to quantize gravity are simply wasting their time (since, according to Jacobson, gravity might not be a fundamental force, but merely a kind of thermodynamic quantum noise). Recently Verlinde has been writing [12] to add extra weight to Jacobson's argument (he claimed that even Newton's laws of motion follow on from a kind of thermodynamical logic).

How watertight is Jacobson's argument experimentally speaking? How much of gravity is really just thermodynamics? The derivation involves two crucial relationships we believe to be true, but which, at present, have no experimental evidence to support them. First of all, the relationship between entropy and area has never been tested. Initially argued within black hole thermodynamics by Bekenstein, the relationship has been elevated to a universally-held principle (i.e., the Holographic principle) by Susskind. Although this principle is supported by a host of other theoretical ideas (including quantum entanglement, the logic I used in my book [13] to explain why entropy is proportional to area), it has never been experimentally tested. Secondly, the Unruh-Davies beautiful formula linking acceleration to temperature requires accelerations far beyond what can presently be achieved in order to generate enough detectable heat (though see the recent proposal in [14]). 


\section{Discussion and Conclusions}

Now, I would like to leave the arena of physics and speculate a bit. A strong interplay between quantum physics and information was first properly pointed out by Everett in his "relative state" interpretation of quantum physics [15]. His motivation came from cosmology, namely the fact that the universe as a whole, and if described quantum mechanically, cannot have an external observer performing measurements. In that sense, the measurement process is simply seen as creation of correlations (as quantified by mutual information in Everett's analysis) between different subsystems in the universe. Everett, however, did not consider the role of information in general relativity, and contrasted it with the role it plays in quantum physics.

If both quantum physics and general theory of relativity have information theoretic underpinnings, perhaps this offers us a way to bring them closer to each other. Of course, this logic would seem to be contrary to that of my own field of quantum computation. This is because quantum computing follows Landauer's dictum [16] that "information is physical". According to this, the laws of physics dictate the laws of information processing (which is why quantum information processing is different to classical). But the above way of arguing that information underlies both quantum physics and relativity goes in the exact opposite direction and puts information before physics ("it from bit" as Wheeler suggested [3]). And putting information before physics is difficult to do, simply because information would have to obey some rules, some axioms that would come prior to the laws of physics. It is hard to imagine where such axioms for information would come from, if not a deeper law of physics.

I do not think that this is a problem, however. The fact that information might be behind most of quantum physics and general relativity, could be telling us which features would survive in the new physical theory when (or if) it comes along. When Einstein was developing relativity, he was in a similar position. He had to decide which features of Newtonian mechanics and Maxwell's electromagnetism to leave intact (he could not keep them all as the Michelson and Morley experiment implied that the speed of light was independent of the reference frame). Einstein decided to keep the principle of invariance of the laws of physics in different reference frames (from Newton) and the upper bound on speed of propagation given by the speed of light (from Maxwell). The resulting two postulates led to the special theory of relativity. We are now asking which of the aspects of quantum physics and general relativity ought to be kept in the foundations of the new unified theory. The answer might be to keep those that have the strongest information flavor. This would suggest that states of physical systems should still be thought of as catalogues of information (so, yes, entanglement would be here to stay) and that entropy is indeed proportional to area. Other things might have to go. Only time will tell.

\section{Acknowledgments}

The author's research is supported by the National Research Foundation and Ministry of Education in Singapore and the John Templeton Foundation. The author is a fellow of Wolfson College, Oxford. 


\section{References}

1. Chandrasekhar, S. Newton's Principia for the Common Reader; Oxford University Press: New York, NY, USA, 1995.

2. Wald, R.M. General Relativity, 1st ed.; University of Chicago Press: Chicago, IL, USA, 1984.

3. Wheeler, J.A. Information, Physics, Quantum: The Search for Links. In Complexity, Entropy and the Physic of Information; Zurek, W.H., Ed.; Westview Press: Boulder, CO, USA, 1990.

4. Kibble, T.W.B. Geometrization of quantum mechanics. Comm. Math. Phys. 1979, 65, 189-201.

5. Schrödinger, E. The Present Situation in Quantum Mechanics. In Quantum Theory and Measurement; Wheeler, J.A., Zurek, W.H., Eds.; Princeton University Press: New Jersey, NJ, USA, 1983.

6. Einstein, A.; Podolsky, B.; Rosen, N. Can quantum-mechanical description of physical reality be considered complete? Phys. Rev. 1935, 47, 777-780.

7. Bell, J.S. On the einstein podolsky rosen paradox. Physics 1964, 1, 195-200.

8. Bekenstein, J. Black holes and the second law. Nuovo Cim. Lett. 1972, 4, 737-740.

9. Hawking, S.W. Particle creation by black holes. Comm. Math. Phys. 1975, 43, 199-220.

10. Jacobson, T. Thermodynamics of spacetime: The einstein equation of state. Phys. Rev. Lett. 1995, $75,1260-1263$.

11. Unruh, W.G. Notes on black-hole evaporation. Phys. Rev. D 1976, 14, 870R-892R.

12. Verlinde. E.P. On the origin of gravity and the Laws of Newton. J. High Energy Phys. 2011, 4, 029.

13. Vedral, V. Decoding Reality: The Universe as Quantum Information; Oxford University Press: Oxford, UK, 2010.

14. Martın-Martınez, E.; Dragan, A.R.; Mann, B.; Fuentes, I. Berry phase quantum thermometer. Available online: http://arxiv.org/pdf/1112.3530.pdf (accessed on 10 May 2012).

15. Everett, H. The Theory of the Universal Wavefunction. In The Many-Worlds Interpretation of Quantum Mechanics; DeWitt, B., Graham, R.N., Eds.; Princeton University Press: Princeton, NJ, USA, 1973; pp. 3-140.

16. Landauer, R. Information is Physical. In Physics and Computation, 1992 Workshop, Dallas, TX, USA, 2-4 October 1992; pp. 1-3.

(C) 2012 by the authors; licensee MDPI, Basel, Switzerland. This article is an open access article distributed under the terms and conditions of the Creative Commons Attribution license (http://creativecommons.org/licenses/by/3.0/). 